\title{
Prediction of maternal and neonatal adverse outcomes in pregnant women treated for hypothyroidism
}

\author{
Maria K. Poulasouchidou, Dimitrios G. Goulis, Pavlos Poulakos, Gesthimani Mintziori, \\ Apostolos Athanasiadis, Grigorios Grimbizis, Basil C. Tarlatzis
}

Unit of Reproductive Endocrinology, First Department of Obstetrics and Gynecology, Medical School, Aristotle University of Thessaloniki, Greece

\begin{abstract}
OBJECTIVE: To examine whether the concentrations of maternal serum TSH and free thyroxine (f $\left.T_{4}\right)$ through pregnancy, the presence of thyroid autoimmunity (TAI) or the dose of levo-thyroxine $\left(\mathbf{L T}_{4}\right)$ replacement can predict the occurrence of maternal or fetal/neonatal complications in pregnant women treated for maternal hypothyroidism. DESIGN: The study included 92 women with singleton pregnancies and primary hypothyroidism on $\mathrm{LT}_{4}$ replacement. Maternal serum $\mathrm{TSH}_{\text {, }} \mathrm{fT}_{4}$, thyroid auto-antibodies and doses of $\mathrm{LT}_{4}$ were monitored throughout pregnancy. All maternal and fetal/neonatal complications were recorded. RESULTS: The overall prevalence of maternal and neonatal complications was $24.1 \%$. Neither maternal $\mathrm{TSH} / \mathrm{fT}_{4}$ concentrations, presence of TAI nor dose of $\mathrm{LT}_{4}$ could predict the occurrence of complications. Pre-pregnancy body mass index (BMI) was higher in women who developed maternal complications [odds ratio $(O R) 1.3,95 \%$ confidence interval $(C I) 1.1-1.5, p=0.007)$ and gestational week at delivery was lower in pregnancies complicated by neonatal (OR 0.5, 95\% CI 0.3-0.8, $p=0.001$ ) or any type of complications (OR $0.6,95 \%$ CI $0.4-0.9, p=0.008)$. CONCLUSIONS: The occurrence of maternal or fetal/neonatal complications in pregnant women treated for hypothyroidism cannot be predicted by maternal TSH/fT $\mathrm{T}_{4}$ through pregnancy, presence of TAI or dose of $\mathrm{LT}_{4}$ replacement.
\end{abstract}

Key words: Adverse pregnancy outcomes, Hypothyroidism, Levo-thyroxine, Thyroid autoimmunity, Thyroid-stimulating hormone

Address for correspondence:

Assistant Professor Dimitrios G. Goulis, Unit of Reproductive Endocrinology, First Department of Obstetrics and Gynecology, "Papageorgiou" General Hospital, Ring Road, 54601 Nea Efkarpia, Thessaloniki, Greece,

Tel: + 2310 693131, Fax: +2310991510,

e-mail: dimitrios.goulis@otenet.gr

Received 01-04-12, Revised 15-06-12, Accepted 07-07-12

\section{INTRODUCTION}

Primary hypothyroidism constitutes the main cause of thyroid dysfunction among women of reproductive age. ${ }^{1}$ In addition, thyroid autoimmunity (TAI), defined as the presence of auto-antibodies against thyroglobulin (TgAb) and/or thyroid peroxidase (TPOAb), has 
been estimated to affect $5-20 \%$ of childbearing age women. ${ }^{2}$ There is evidence that pregnancy exerts a stressful impact on the thyroid gland, resulting in alterations of iodine economy and thyroid function. ${ }^{3,4}$ It is in this context that primary hypothyroidism is commonly diagnosed in pregnant women, with an estimated prevalence of $0.4 \%$ and $3 \%$ for clinical and subclinical hypothyroidism, respectively. ${ }^{5}$ Although titers of thyroid auto-antibodies have been found to decrease by $50 \%$ during pregnancy, ${ }^{6}$ it is estimated that $16 \%$ of euthyroid pregnant women with TAI will develop hypothyroidism during pregnancy. ${ }^{7}$

Both hypothyroidism and TAI have been associated with adverse pregnancy outcomes. Untreated clinical hypothyroidism in pregnancy has been associated with an increased risk of congenital abnormalities, miscarriage, premature birth, low birth weight, perinatal mortality and gestational hypertension. ${ }^{5}$ Untreated subclinical hypothyroidism has been associated with an increased risk of perinatal mortality and pre-eclampsia. ${ }^{8}$ Finally, TAI per se has been associated with an increased risk of premature birth, miscarriage and perinatal mortality. ${ }^{8,9}$ Though causality remains unclear, the association of TAI with adverse pregnancy outcomes has been attributed to impaired thyroid function in the presence of TAI, co-existence of other autoimmune diseases and direct action of thyroid auto-antibodies on the reproductive tissues, such as ovary, endometrium and placenta. ${ }^{10}$ Despite this association between hypothyroidism/ TAI and adverse pregnancy outcome, little is known about the thyroid parameters that can predict such an outcome. ${ }^{11,12}$

The aim of this prospective study was to examine whether the concentrations of maternal serum TSH and $\mathrm{fT}_{4}$ through pregnancy, the presence of TAI or the dose of levo-thyroxine $\left(\mathrm{LT}_{4}\right)$ replacement can predict the occurrence of maternal or fetal/neonatal complications in pregnant women treated for hypothyroidism.

\section{PATIENTS AND METHODS}

\section{Type of study type}

A prospective, cohort, clinical study.

\section{Setting}

Patients were recruited from the outpatient clinics of the Unit of Reproductive Endocrinology, First Department of Obstetrics and Gynecology, Aristotle University of Thessaloniki, Greece, which is a tertiary, referral center for women with endocrine and metabolic complications of pregnancy. The study protocol was approved by the Bioethics Committee, Aristotle University of Thessaloniki.

\section{Patients}

The study included 92 consecutive cases of pregnant women with primary hypothyroidism during the period January 2007 - December 2010. Inclusion criteria were: i) primary hypothyroidism diagnosed before or during index pregnancy, on $\mathrm{LT}_{4}$ replacement, and ii) singleton pregnancy. Exclusion criterion was presence of co-morbidities, such as type 1 or type 2 diabetes mellitus or gestational diabetes (GDM) in the index pregnancy, as they might have introduced bias. Possible subsequent pregnancies in the same women during the study period were also excluded so that each woman participated in the data analysis only once.

\section{Study parameters}

In all pregnant women, the following parameters were recorded during their first antenatal evaluation: type of conception (spontaneous/assisted), maternal age at conception, height, pre-pregnancy weight, body mass index (BMI), current smoking status (yes/no), parity (categorical: $0,1, \geq 2$ ), history of spontaneous miscarriages (categorical: $0,1, \geq 2$ ), history of recurrent miscarriages (yes/no), history of preterm deliveries (yes/no) and family history of thyroid dysfunction (yes/no). Weight gain was recorded throughout the index pregnancy. The main hormonal parameters evaluated were: i) maternal serum TSH and free thyroxine $\left(\mathrm{fT}_{4}\right)$, ii) thyroid auto-antibodies (TPOAb and $\mathrm{TgAb}$ ) and iii) doses of $\mathrm{LT}_{4}$ replacement.

\section{Follow-up protocol}

Women were followed up every four to six weeks until delivery. In those with hypothyroidism diagnosed during pregnancy, $\mathrm{LT}_{4}$ substitution was initiated at first antenatal visit. Maternal serum $\mathrm{TSH}$ and $\mathrm{fT}_{4}$ evaluation was performed at four-week intervals after 
$\mathrm{LT}_{4}$ initiation or dose adjustment and at least once in each pregnancy trimester. All women were instructed to have a postnatal thyroid function evaluation at six weeks after delivery.

\section{Assays}

Serum samples were analyzed at the biochemical laboratory of the "Papageorgiou" General Hospital, Thessaloniki, Greece. A third generation chemiluminescent immunometric assay (Immulite 2000, Siemens, Germany) was employed for serum TSH (reference range $0.4-4 \mu \mathrm{IU} / \mathrm{ml}$ ). Serum $\mathrm{fT}_{4}$ was measured using chemiluminescent competitive immunoassay (Immulite 2000, Siemens, Germany) with a reference range of $0.89-1.76 \mathrm{ng} / \mathrm{dl}$. The intra-assay coefficient of variation $(\mathrm{CV})$ for $\mathrm{TSH}$ and $\mathrm{fT}_{4}$ was $3.9 \%$ and $4.8 \%$, respectively. Determination of $\mathrm{TgAb}$ and TPOAb was made by the Micro-Enzyme Immunoassay (ELISA) method (Varelisa, EliA, Sweden Diagnostics, GmbH, Freiburg, Germany).

\section{Primary and secondary outcomes}

The primary outcomes of the study were the occurrence of i) any type of complication (combined outcome), ii) maternal complications and iii) neonatal complications. Maternal complications included pregnancy-induced hypertension, pre-eclampsia, placental abruption, poly-hydramnion, oligoamnion and hyperemesis gravidarum. Though the latter has been strongly associated with overt hyperthyroidism, it can also be a result of high hCG concentrations at the end of the first trimester and its homology to TSH. Neonatal complications included jaundice, tachypnoea, respiratory distress syndrome, hypothyroidism and inguinal hernia. Secondary outcomes were the occurrence of: i) pregnancy loss (any abortion or intra-uterine death from clinical pregnancy to delivery) and ii) miscarriage (any abortion from clinical pregnancy until gestational week 22).

\section{Statistical analysis}

The Kolmogorov-Smirnov and Shapiro-Wilks tests were applied to verify the normal distribution of continuous variables. Continuous variables were expressed as mean \pm standard deviation (SD), if normally distributed, and as median (minimum maximum), if not normally distributed. Categorical variables were expressed as absolute numbers and percentages. The t-Student or Mann-Whitney tests were used for comparison of the means in continuous variables between two groups. The chi-square test was used for comparison of ratios in categorical variables. Univariate and multivariate logistic regression models were built to model the relationship between study outcomes and one or more explanatory variables, respectively. Statistical significance level was set at $p<0.05$. All analyses were performed using SPSS 17 (SPSS Inc, Chicago, Ill, USA).

\section{RESULTS}

\section{Obstetrical characteristics}

The mean age of the studied women was $32 \pm 4$ years with a pre-gestational BMI of $23(17-39) \mathrm{kg} /$ $\mathrm{m}^{2}$. Total weight gain during pregnancy was $13(4-$ $30) \mathrm{kg}$. Forty-eight percent were nulliparous and $13 \%$ became pregnant after in vitro fertilization (IVF). A history of spontaneous miscarriage, recurrent miscarriage or preterm delivery was recorded in $33 \%$, $17.6 \%$ and $9.8 \%$ of the studied women, respectively. The mean birth weight was $3086 \pm 444 \mathrm{~g}$.

\section{Thyroid characteristics}

Primary hypothyroidism was diagnosed either before $(\mathrm{n}=78,84.8 \%)$ or during $(\mathrm{n}=14,15.2 \%)$ index pregnancy. In nine out of fourteen women with newly diagnosed hypothyroidism, the diagnosis was made during the first trimester (mean $8.2 \pm 2.4$ gestational week), in three during the second trimester [median $20(19-21)$ gestational week] and in two during the third trimester of pregnancy [median 33 (31.4 - 34.1) gestational week]. Women with hypothyroidism diagnosed during the index pregnancy did not differ significantly in age ( $32 \pm 4$ vs. $32 \pm 5$ years, $p=0.492)$, pre-gestational BMI [24.1 (20.6 - 28.6) vs. $22.7(17.2-39.3) \mathrm{kg} / \mathrm{m}^{2}, p=0.486$ ] or weight gain during pregnancy [16 $(9-22)$ vs. $13(4-30) \mathrm{kg}, p$ $=0.097$ in comparison to women with pre-existing hypothyroidism. TAI was diagnosed in $65 \%$ of the total cohort of women.

\section{Primary outcomes}

Study outcomes are demonstrated in Figure 1. Predictive factors for primary study outcomes (maternal, neonatal and combined complications) are presented in Table 1. Presence of TAI did not differ 
significantly between the women who developed or did not any type of complication. The same was true for maternal $\mathrm{fT}_{4}$ concentrations and $\mathrm{LT}_{4}$ doses, either before or throughout pregnancy. On the other hand, pre-gestational TSH was marginally higher in women who developed any type of complication (combined outcome) as compared to women with uncomplicated pregnancies. In a similar way, a history of preterm delivery was significantly more common in women who developed any type of complication (combined outcome) as compared to women with uncomplicated pregnancies. Finally, pre-gestational BMI was significantly higher in women who developed maternal complications and gestational week at delivery was lower in pregnancies that resulted in neonatal or any type of complication (combined outcome), as compared to women with uncomplicated pregnancies. All main study outcomes remained unchanged after a sensitivity analysis that excluded women that underwent IVF (12 women, $13 \%$ of the studied sample). The only exception was pre-gestational TSH for the combined outcome, which reached statistical significance [4.55 $(1.50-9.24)$ vs. $1.67(0.03-18.10), p=0.039]$.

\section{Secondary outcomes}

Predictive factors for secondary study outcomes (pregnancy loss and spontaneous miscarriage rates) are presented in Table 2 .

\section{Logistic regression models}

In univariate models (Table 3 ), history of preterm deliveries, pre-gestational dose of $\mathrm{LT}_{4}$ and gestational week at delivery were significantly different in women who developed any type of complication. In multivariate models, only dose of $\mathrm{LT}_{4}$ retained its significance. Pre-gestational BMI was significantly higher in women who developed maternal complication, but it did not retain its significance in the multivariate model. Finally, gestational week at delivery was significantly lower in women who developed neonatal complication, but it did not retain its significance in the multivariate model.

\section{DISCUSSION}

Aim and main findings. This prospective study of pregnancies treated for maternal hypothyroidism

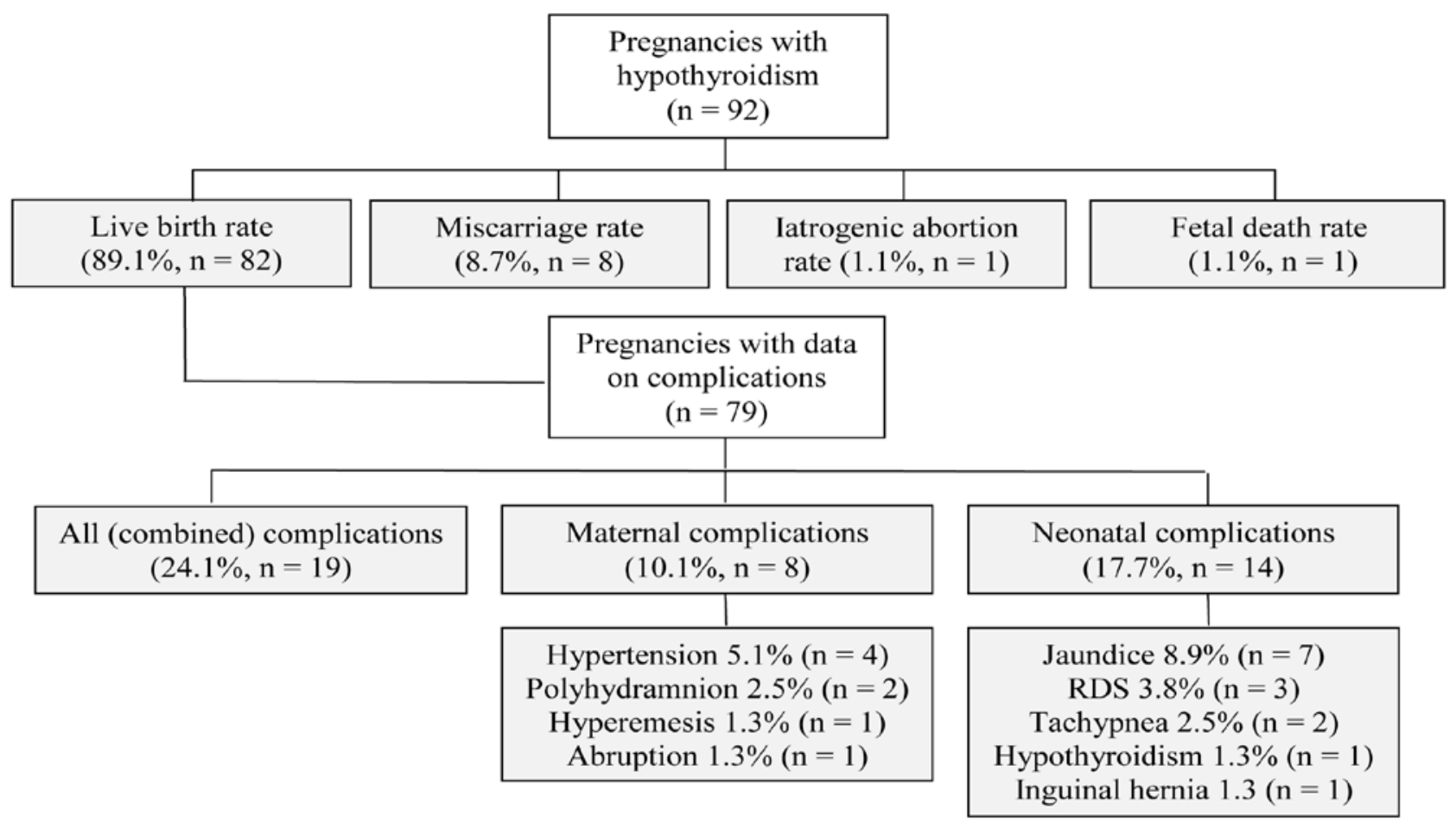

Figure 1. Study outcomes. 
Table 1. Predictive factors for primary study outcomes: all (combined outcome), maternal and neonatal complications in pregnant women treated for hypothyroidism

\begin{tabular}{|c|c|c|c|}
\hline Predictive parameters & Complications present & Complications absent & p value \\
\hline \multicolumn{4}{|c|}{ All complications (combined) } \\
\hline $\mathrm{N}(\%)$ & $19(24.1)$ & $60(75.9)$ & - \\
\hline Maternal age (years) & $33 \pm 5$ & $31 \pm 4$ & 0.257 \\
\hline BMI, pre-gestation $\left(\mathrm{kg} / \mathrm{m}^{2}\right)$ & $24.5(17.3-39.3)$ & $22.5(17.2-35.1)$ & 0.279 \\
\hline Weight gain $(\mathrm{kg})$ & $13(10-25)$ & $13(4-30)$ & 0.121 \\
\hline Conception after IVF (\%) & 10.5 & 13.3 & 1.000 \\
\hline Gestation week at delivery (week) & $37.5(34-39)$ & $38(34-41)$ & 0.027 \\
\hline Hx of preterm delivery $(\%)$ & 30 & 3 & 0.034 \\
\hline Hx of spontaneous miscarriages (\%) & 21.1 & 27.8 & 0.763 \\
\hline TAI $(\%)$ & 44.7 & 51.7 & 0.744 \\
\hline TSH, pre-gestation (mU/ml) & $3.92(1.27-9.24)$ & $1.69(0.03-18.10)$ & 0.066 \\
\hline first trimester & $3.65(0.36-26.5)$ & $3.24(0.01-60.00)$ & 0.655 \\
\hline second trimester & $2.12(0.41-6.5)$ & $1.97(0.01-41.00)$ & 0.408 \\
\hline third trimester & $1.43(0.11-3.04)$ & $1.24(0.05-5.78)$ & 0.750 \\
\hline $\mathrm{fT}_{4}$, pre-gestation $(\mathrm{ng} / \mathrm{l})$ & $1.10(1.04-1.42)$ & $1.39(0.85-1.90)$ & 0.080 \\
\hline first trimester & $1.07(0.92-1.69)$ & $1.28(0.63-2.30)$ & 0.627 \\
\hline second trimester & $0.98(0.75-1.42)$ & $1.08(0.71-3.44)$ & 0.571 \\
\hline third trimester & $1.03 \pm 0.13$ & $1.13 \pm 0.26$ & 0.276 \\
\hline $\mathrm{LT}_{4}$ dose, pre-gestation $(\mu \mathrm{g} / \mathrm{d})$ & $0(0-75)$ & $75(0-150)$ & 0.013 \\
\hline first trimester & $50(0-150)$ & $75(0-175)$ & 0.279 \\
\hline second trimester & $86(0-150)$ & $100(0-175)$ & 0.339 \\
\hline third trimester & $88 \pm 50$ & $106 \pm 42$ & 0.190 \\
\hline \multicolumn{4}{|c|}{ Maternal complications } \\
\hline $\mathrm{N}(\%)$ & $8(10.1)$ & $71(89.9)$ & - \\
\hline Maternal age (years) & $33 \pm 6$ & $32 \pm 4$ & 0.276 \\
\hline BMI, pre-gestation $\left(\mathrm{kg} / \mathrm{m}^{2}\right)$ & $30.5(24.5-39.3)$ & $22.5(17.2-35.1)$ & 0.004 \\
\hline Weight gain during pregnancy $(\mathrm{kg})$ & $12(10-22)$ & $13(4-30)$ & 0.697 \\
\hline Conception after IVF (\%) & 25.0 & 11.3 & 0.266 \\
\hline TAI $(\%)$ & 50.0 & 64.4 & 0.619 \\
\hline TSH, pre-gestation (mU/ml) & $3.30(1.27-7.31)$ & $2.18(0.03-18.10)$ & 0.418 \\
\hline first trimester & $1.87(0.36-6.30)$ & $3.36(0.01-60.00)$ & 0.374 \\
\hline second trimester & $2.47(1.05-3.10)$ & $1.98(0.01-41.00)$ & 0.965 \\
\hline third trimester & $1.33(0.70-1.67)$ & $1.34(0.05-5.78)$ & 0.715 \\
\hline $\mathrm{fT}_{4}$, pre-gestation (ng/l) & $1.14 \pm 0.06$ & $1.35 \pm 0.28$ & 0.327 \\
\hline first trimester & $1.21 \pm 0.32$ & $1.26 \pm 0.29$ & 0.740 \\
\hline second trimester & $1.06 \pm 0.27$ & $1.15 \pm 0.39$ & 0.573 \\
\hline third trimester & $0.97(0.86-1.16)$ & $1.07(0.52-1.88)$ & 0.195 \\
\hline $\mathrm{LT}_{4}$ dose, pre-gestation $(\mu \mathrm{g} / \mathrm{d})$ & $50(0-75)$ & $75(0-150)$ & 0.380 \\
\hline first trimester & $81(0-150)$ & $75(0-175)$ & 0.483 \\
\hline second trimester & $100(50-150)$ & $100(0-175)$ & 0.587 \\
\hline third trimester & $102 \pm 47$ & $102 \pm 45$ & 0.963 \\
\hline
\end{tabular}

BMI: body mass index; fT 4 : free thyroxine; Hx: history; IVF: in vitro fertilization; $\mathrm{LT}_{4}$ : levo-thyroxine; TAI: thyroid auto-immunity; TSH: thyroid stimulating hormone. 
Table 1. Predictive factors for primary study outcomes: all (combined outcome), maternal and neonatal complications in pregnant women treated for hypothyroidism (continued)

\begin{tabular}{|c|c|c|c|}
\hline Predictive parameters & Complications present & Complications absent & p value \\
\hline \multicolumn{4}{|c|}{ Neonatal complications } \\
\hline $\mathrm{N}(\%)$ & $14(17.7)$ & $65(82.3)$ & - \\
\hline Maternal age (years) & $33 \pm 5$ & $32 \pm 4$ & 0.381 \\
\hline BMI, pre-gestation $\left(\mathrm{kg} / \mathrm{m}^{2}\right)$ & $23.2(17.3-32.0)$ & $22.9(17.2-39.3)$ & 0.697 \\
\hline Weight gain during pregnancy $(\mathrm{kg})$ & $15(10-25)$ & $13(4-30)$ & 0.268 \\
\hline Conception after IVF (\%) & 7.1 & 14.1 & 0.680 \\
\hline Gestation week at delivery (week) & $36.5(34-39)$ & $38(34-41)$ & 0.004 \\
\hline TAI $(\%)$ & 66.7 & 63.0 & 1.000 \\
\hline TSH, pre-gestation (mU/ml) & $4.50(1.50-9.24)$ & $1.71(0.03-18.10)$ & 0.112 \\
\hline first trimester & $3.70(1.77-26.50)$ & $3.24(0.01-60.00)$ & 0.251 \\
\hline second trimester & $2.96(0.41-6.50)$ & $1.97(0.01-41.00)$ & 0.243 \\
\hline third trimester & $1.43(0.11-3.04)$ & $1.30(0.05-5.78)$ & 0.921 \\
\hline $\mathrm{fT}_{4}$, pre-gestation $(\mathrm{ng} / \mathrm{l})$ & $1.20 \pm 0.19$ & $1.35 \pm 0.28$ & 0.385 \\
\hline first trimester & $1.18(0.92-1.53)$ & $1.27(0.63-2.30)$ & 0.761 \\
\hline second trimester & $1.02 \pm 0.26$ & $1.16 \pm 0.39$ & 0.344 \\
\hline third trimester & $1.00(0.98-1.16)$ & $1.07(0.52-1.88)$ & 0.427 \\
\hline $\mathrm{LT}_{4}$ dose, pre-gestation $(\mu \mathrm{g} / \mathrm{d})$ & $0(0-70)$ & $75(0-150)$ & 0.020 \\
\hline first trimester & $60(0-150)$ & $75(0-175)$ & 0.385 \\
\hline second trimester & $86(0-150)$ & $100(0-175)$ & 0.381 \\
\hline third trimester & $81(0-175)$ & $100(0-200)$ & 0.346 \\
\hline
\end{tabular}

BMI: body mass index; $\mathrm{fT}_{4}$ : free thyroxine; Hx: history; IVF: in vitro fertilization; $\mathrm{LT}_{4}$ : levo-thyroxine; TAI: thyroid auto-immunity; TSH: thyroid stimulating hormone.

aimed to examine whether thyroid parameters (concentrations of serum $\mathrm{TSH}$ and $\mathrm{fT}_{4}$, presence of TAI and dose of $\mathrm{LT}_{4}$ replacement), recorded before and throughout pregnancy, can predict the occurrence of maternal or fetal/neonatal complications. None of the evaluated thyroid parameters was significantly associated with the occurrence of any type of complication. On the other hand, pre-gestational BMI and history of preterm deliveries were significantly associated with an increased risk of maternal and combined maternal/ neonatal complications, respectively. In addition, the risk of neonatal complications increased significantly with decreasing gestation week at delivery.

Interpretation of the findings. The results of this study indicate that in women with adequately treated hypothyroidism, thyroid parameters do not provide a significant contribution in predicting the occurrence of maternal or fetal/neonatal complications.

Pre-gestational TSH was marginally higher in pregnancies affected by both maternal and/or neonatal complications (combined outcome) in comparison to unaffected pregnancies. In this setting, it seems plausible to state that pre-gestational TSH concentrations are stronger predictors of pregnancy outcome as compared to TSH concentrations during pregnancy. The latter are a result of the uniform application of the Endocrine Society's guidelines ${ }^{13}$ according to which first trimester TSH concentrations should be $<2.5 \mathrm{mIU} / \mathrm{ml}$. As a direct consequence, confirmation of pregnancy resulted, in the majority of cases, in an increase in $\mathrm{LT}_{4}$ doses and a decrease in maternal TSH concentrations. Nevertheless, even pre-gestational TSH is far from being significant in the prediction of serious adverse outcomes, such as pregnancy loss or miscarriage.

Comparison with the literature. Two studies aimed to indicate thyroid parameters that could predict the outcome of pregnancies complicated by hypothyroidism. In the first study, Ashoor et $\mathrm{al}^{11}$ demonstrated 
Table 2. Predictive factors for secondary study outcomes: pregnancy loss and spontaneous miscarriage rates in pregnant women treated for hypothyroidism

\begin{tabular}{|c|c|c|c|}
\hline Predictive parameters & Complications present & Complications absent & p value \\
\hline \multicolumn{4}{|c|}{ Pregnancy loss } \\
\hline $\mathrm{N}(\%)$ & $10(10.9)$ & $82(89.1)$ & - \\
\hline Maternal age (years) & $32(29-42)$ & $32(22-41)$ & 0.970 \\
\hline BMI, pre-gestation $\left(\mathrm{kg} / \mathrm{m}^{2}\right)$ & $24.2(18.0-32.4)$ & $22.9(17.2-39.3)$ & 0.590 \\
\hline Conception after IVF (\%) & 22.0 & 12.2 & 0.614 \\
\hline Hx of spontaneous miscarriages (\%) & 44.0 & 25.7 & 0.450 \\
\hline Hx of preterm delivery $(\%)$ & 14.3 & 9.1 & 0.538 \\
\hline TAI $(\%)$ & 50.0 & 52.4 & 1.000 \\
\hline TSH, pre-gestation (mU/ml) & $4.50(1.06-6.70)$ & $2.76(0.03-18.10)$ & 0.233 \\
\hline first trimester & $2.36(0.80-13.40)$ & $3.48(0.01-60.00)$ & 0.246 \\
\hline second trimester & $\mathrm{N} / \mathrm{A}$ & $2.00(0.01-21.00)$ & $\mathrm{N} / \mathrm{A}$ \\
\hline third trimester & $\mathrm{N} / \mathrm{A}$ & $1.31(0.05-5.78)$ & $\mathrm{N} / \mathrm{A}$ \\
\hline $\mathrm{fT}_{4}$, pre-gestation (ng/l) & $1.36 \pm 0.17$ & $1.35 \pm 0.29$ & 0.939 \\
\hline first trimester & $1.36(1.23-3.10)$ & $1.27(0.63-3.30)$ & 0.075 \\
\hline second trimester & $\mathrm{N} / \mathrm{A}$ & $1.08(0.71-3.44)$ & - \\
\hline third trimester & $\mathrm{N} / \mathrm{A}$ & $1.07(0.52-1.88)$ & - \\
\hline $\mathrm{LT}_{4}$ dose, pre-gestation $(\mu \mathrm{g} / \mathrm{d})$ & $62(0-125)$ & $75(0-150)$ & 0.913 \\
\hline first trimester & $100(50-125)$ & $75(0-175)$ & 0.418 \\
\hline second trimester & N/A & $100(0-175)$ & - \\
\hline third trimester & $\mathrm{N} / \mathrm{A}$ & $100 \pm 44$ & - \\
\hline \multicolumn{4}{|c|}{ Miscarriage } \\
\hline $\mathrm{N}(\%)$ & $8(8.7)$ & $84(91.3)$ & - \\
\hline Maternal age (years) & $33 \pm 4$ & $32 \pm 4$ & 0.608 \\
\hline BMI, pre-gestation (kg/m2) & $19.1(18.0-32.3)$ & $23.0(17.2-39.3)$ & 0.462 \\
\hline Conception after IVF (\%) & 25 & 12 & 0.279 \\
\hline Hx of spontaneous miscarriages (\%) & 50.0 & 25.0 & 0.206 \\
\hline Hx of recurrent miscarriages $(\%)$ & 25.0 & 17.1 & 0.629 \\
\hline Hx of preterm delivery $(\%)$ & 16.7 & 8.9 & 0.480 \\
\hline TAI $(\%)$ & 62.5 & 51.2 & 0.617 \\
\hline TSH, pre-gestation (mU/ml) & $3.66(1.06-6.70)$ & $2.90(0.03-18.10)$ & 0.510 \\
\hline first trimester & $2.36(0.80-4.62)$ & $3.36(0.01-60.00)$ & 0.171 \\
\hline fT4, pre-gestation (ng/l) & $1.40 \pm 0.16$ & $1.34 \pm 0.29$ & 0.666 \\
\hline first trimester & $1.44(1.23-3.10)$ & $1.27(0.63-2.30)$ & 0.044 \\
\hline LT4, dose pre-gestation $(\mu \mathrm{g} / \mathrm{d})$ & $62(0-125)$ & $75(0-150)$ & 0.769 \\
\hline first trimester & $100(50-125)$ & $75(0-175)$ & 0.265 \\
\hline
\end{tabular}

BMI: body mass index; $\mathrm{fT}_{4}$ : free thyroxine; Hx: history; IVF: in vitro fertilization; $\mathrm{LT}_{4}$ : levo-thyroxine; N/A: not applicable; TAI: thyroid auto-immunity; TSH: thyroid stimulating hormone.

a significant association between low maternal $\mathrm{fT}_{4}$ during the first trimester and fetal loss, in pregnancies complicated by subclinical hypothyroidism. In the second study by Benhadi et al, ${ }^{12}$ maternal TSH was significantly associated with the occurrence of pregnancy loss in pregnant women without overt hypothyroidism. No significant association was reported between maternal $\mathrm{fT}_{4}$ and pregnancy loss. ${ }^{12}$ 
Table 3. Logistic regression models of maternal and neonatal complications in pregnant women treated for hypothyroidism

\begin{tabular}{|c|c|c|c|c|c|c|}
\hline \multirow[b]{2}{*}{ Predictive parameters } & \multicolumn{3}{|c|}{ Univariate models } & \multicolumn{3}{|c|}{ Multivariate models } \\
\hline & OR & $95 \% \mathrm{CI}$ & p value & OR & $95 \% \mathrm{CI}$ & p value \\
\hline \multicolumn{7}{|c|}{ All complications (combined) } \\
\hline History of preterm delivery & 13.7 & $1.2-152.1$ & 0.033 & - & - & - \\
\hline TSH, pre-gestation & 1.1 & $0.9-1.4$ & 0.257 & - & - & - \\
\hline $\mathrm{fT}_{4}$, pre-gestation & 0.0 & $0.0-1.8$ & 0.094 & - & - & - \\
\hline $\mathrm{LT}_{4}$ dose, pre-gestation & 1.0 & $1.0-1.0$ & 0.028 & 1.0 & $1.0-1.0$ & 0.018 \\
\hline Gestational week at delivery & 0.6 & $0.4-0.9$ & 0.008 & 0.6 & $0.4-1.1$ & 0.074 \\
\hline \multicolumn{7}{|c|}{ Maternal complications } \\
\hline BMI, pre-gestation & 1.3 & $1.1-1.5$ & 0.007 & 1.4 & $0.9-2.0$ & 0.119 \\
\hline $\mathrm{fT}_{4}$, third trimester & 0.0 & $0.0-6.4$ & 0.163 & 0.0 & $0.0-19.7$ & 0.096 \\
\hline \multicolumn{7}{|c|}{ Neonatal complications } \\
\hline TSH, pre-gestation & 1.1 & $0.9-1.4$ & 0.271 & - & - & - \\
\hline $\mathrm{LT}_{4}$ dose, pre-gestation & 1.0 & $0.9-1.0$ & 0.051 & 1.0 & $0.9-1.0$ & 0.038 \\
\hline Gestational week at delivery & 0.5 & $0.3-0.8$ & 0.001 & 0.3 & $0.1-0.9$ & 0.024 \\
\hline
\end{tabular}

BMI: body mass index; $\mathrm{CI}$ : confidence interval; $\mathrm{fT}_{4}$ : free thyroxine; $\mathrm{Hx}$ : history; $\mathrm{LT}_{4}$ : levo-thyroxine; OR: odds ratio; $\mathrm{TSH}$ : thyroid stimulating hormone.

Our study was designed to indicate predictors for pregnancy complications, whereas fetal loss was only a secondary aim.

Two recently published meta-analyses conclude that presence of TAI is associated with an increased risk of miscarriage, perinatal mortality and preterm delivery, ${ }^{8,9}$ whereas no association is reported for maternal or neonatal adverse outcomes. ${ }^{8}$ In our study of women with treated hypothyroidism, presence of TAI was not significantly associated either with increased risk of miscarriage or with the occurrence of maternal or neonatal complications. A possible explanation may be found in the study of Negro et al, ${ }^{14}$ which demonstrated that administration of $\mathrm{LT}_{4}$ in euthyroid pregnant women with positive TPOAb decreases the risk for miscarriage and preterm deliveries.

This study did not use a control group; nevertheless, it allowed for a direct comparison of complications between pregnant women with or without hypothyroidism. It reported a low incidence of maternal and fetal/neonatal complications. Abalovich et $\mathrm{al}^{15}$ demonstrated that adequate $\mathrm{LT}_{4}$ replacement during pregnancy minimizes the risk of miscarriage and preterm delivery. In a study of Tan et al, ${ }^{16}$ pregnancy-related complications of the mother and the fetus/neonate were compared between 419 women with treated hypothyroidism and 20,080 without hypothyroidism. The study concluded that treated hypothyroidism was not associated with an increased risk of maternal or neonatal complications. Similar results were reported in a study by Matalon et al. ${ }^{17}$

Strengths and limitations. The main strength of this study is its prospective nature that allowed for multiple thyroid function determinations throughout pregnancy. The main limitation is its rather small sample size. If a larger sample of women had been involved, selected parameters (e.g. pre-gestational TSH concentration) might have proved to be significant predictors of the outcome. Nevertheless, the study has been sufficiently powered to demonstrate the significance of parameters, such as pre-pregnancy BMI and gestational week at delivery. Thus, even if specific thyroid parameters could predict the outcome of a pregnancy, its contribution would most likely be weak.

Conclusions. This study on pregnant women treated for hypothyroidism: 1) found no evidence that maternal TSH/fT $\mathrm{T}_{4}$ through pregnancy, presence of TAI or dose of $\mathrm{LT}_{4}$ replacement can predict the occurrence of maternal or fetal/neonatal complications; 2) found 
evidence that pre-gestational maternal TSH concentrations tend to be higher in pregnancies affected by complications in comparison to unaffected ones; 3 ) found evidence that pre-gestational BMI, history of preterm deliveries and gestation week at delivery are significantly associated with an increased risk of maternal/neonatal complications. Large prospective studies are needed in order to elucidate the complex interplay between thyroid axis or TAI and pregnancy.

This work is part of a thesis for the M.Sc. degree "Medical Research Methodology", Medical School, Aristotle University of Thessaloniki, Greece.

\section{REFERENCES}

1. Mandel SJ, 2004 Hypothyroidism and chronic autoimmune thyroiditis in the pregnant state: maternal aspects. Best Pract Res Clin Endocrinol Metab 18: 213-224.

2. Chen L, Hu R, 2011 Thyroid autoimmunity and miscarriage: a meta-analysis. Clin Endocrinol (Oxf) 74: 513-519.

3. Glinoer D, 1997 The regulation of thyroid function in pregnancy: pathways of endocrine adaptation from physiology to pathology. Endocr Rev 18: 404-433.

4. Glinoer D, 2004 The regulation of thyroid function during normal pregnancy: importance of the iodine nutrition status. Best Pract Res Clin Endocrinol Metab 18: 133-152.

5. Krassas GE, Poppe K, Glinoer D, 2010 Thyroid function and human reproductive health. Endocr Rev 31: 702-755.

6. Smyth PP, Wijeyaratne CN, Kaluarachi WN, et al, 2005 Sequential studies on thyroid antibodies during pregnancy. Thyroid 15: 474-477.

7. Glinoer D, Riahi M, Grun JP, Kinthaert J, 1994 Risk of subclinical hypothyroidism in pregnant women with asymptomatic autoimmune thyroid disorders. J Clin Endocrinol Metab 79: 197-204.

8. van den BE, Vissenberg R, Land JA, et al, 2011 Significance of (sub)clinical thyroid dysfunction and thyroid autoimmunity before conception and in early pregnancy: a systematic review. Hum Reprod Update 17: 605-619.

9. Thangaratinam S, Tan A, Knox E, Kilby MD, Franklyn J, Coomarasamy A, 2011 Association between thyroid autoantibodies and miscarriage and preterm birth: metaanalysis of evidence. BMJ 342: d2616.

10. Twig G, Shinab A, Amitala H, Shoenfeld Y, 2011 Pathogenesis of infertility and recurrent pregnancy loss in thyroid autoimmunity. J Autoimmun 38: J275-J281.

11. Ashoor G, Maiz N, Rotas M, Jawdat F, Nicolaides KH, 2010 Maternal thyroid function at 11 to 13 weeks of gestation and subsequent fetal death. Thyroid 20: 989993.

12. Benhadi N, Wiersinga WM, Reitsma JB, Vrijkotte TG, Bonsel GJ, 2009 Higher maternal TSH levels in pregnancy are associated with increased risk for miscarriage, fetal or neonatal death. Eur J Endocrinol 160: 985-991.

13. Abalovich M, Amino N, Barbour LA, et al, 2007 Management of thyroid dysfunction during pregnancy and postpartum: an Endocrine Society Clinical Practice Guideline. J Clin Endocrinol Metab 92: Suppl 8: 1-47.

14. Negro R, Formoso G, Mangieri T, Pezzarossa A, Dazzi D, Hassan H, 2006 Levothyroxine treatment in euthyroid pregnant women with autoimmune thyroid disease: effects on obstetrical complications. J Clin Endocrinol Metab 91: 2587-2591.

15. Abalovich M, Gutierrez S, Alcaraz G, Maccallini G, Garcia A, Levalle O, 2002 Overt and subclinical hypothyroidism complicating pregnancy. Thyroid 12: 63-68.

16. Tan TO, Cheng YW, Caughey AB, 2006 Are women who are treated for hypothyroidism at risk for pregnancy complications? Am J Obstet Gynecol 194: e1-e3.

17. Matalon S, Sheiner E, Levy A, Mazor M, Wiznitzer A, 2006 Relationship of treated maternal hypothyroidism and perinatal outcome. J Reprod Med 51: 59-63. 